\title{
Discontinuation of antidepressants in people with dementia and neuropsychiatric symptoms (DESEP study): double blind, randomised, parallel group, placebo controlled trial
}

\author{
(c) $(1)(9)$ OPEN ACCESS
}

\author{
Sverre Bergh researcher ${ }^{1}$, Geir Selbæk head of research department ${ }^{12}$, Knut Engedal professor ${ }^{123}$ \\ ${ }^{1}$ Centre for Old Age Psychiatric Research, Innlandet Hospital Trust, N-2312 Ottestad, Norway ; ${ }^{2}$ Norwegian Centre for Dementia Research, Oslo \\ University Hospital, Oslo, Norway ; ${ }^{3}$ University of Oslo, Oslo
}

\begin{abstract}
Objective To determine the effect of discontinuing antidepressant treatment in people with dementia and neuropsychiatric symptoms.

Design Double blind, randomised, parallel group, placebo controlled trial.

Setting Norwegian nursing homes; residents recruited by 16 study centres in Norway from August 2008 to June 2010.

Participants 128 patients with Alzheimer's disease, dementia or vascular dementia, and neuropsychiatric symptoms (but no depressive disorder), who had been prescribed escitalopram, citalopram, sertraline, or paroxetine for three months or more. We excluded patients with severe somatic disease or terminal illness, or who were unable to take tablets or capsules as prescribed.
\end{abstract}

Interventions Antidepressant treatment was discontinued over one week in 63 patients, and continued in 68 patients. We assessed patients at baseline, four, seven, 13, and 25 weeks.

Main outcome measures Primary outcomes were score differences between study groups in the Cornell scale of depression in dementia and the neuropsychiatric inventory (10 item version) after 25 weeks. Secondary outcomes were score differences in the clinical dementia rating scale, unified Parkinson's disease rating scale, quality of life-Alzheimer's disease scale, Lawton and Brody's physical self maintenance scale, and severe impairment battery.

Results Using a linear multilevel model analysis, we found that the discontinued group had significantly higher scores on the Cornell scale after 25 weeks than the continuation group (difference -2.89 (95\% confidence interval -4.76 to -1.02 ); $P=0.003$ ). We saw a similar result in the mean total score for the neuropsychiatric inventory after 25 weeks, but this difference was non-significant $(-5.96$ (-12.35 to 0.44$) ; \mathrm{P}=0.068)$. We confirmed these results by non-response analysis ( $>30 \%$ worsening on the Cornell scale)-significantly more patients worsened in the discontinuation group than in the continuation group (32 (54\%) $v 17$
(29\%); $P=0.006)$. We found no significant differences between the groups for secondary outcomes. Forty seven (37\%) patients withdrew from the study early.

Conclusions Discontinuation of antidepressant treatment in patients with dementia and neuropsychiatric symptoms leads to an increase in depressive symptoms, compared with those patients who continue with treatment.

Trial registration ClinicalTrial.gov NCT00594269, EudraCT 2006-002790-43.

\section{Introduction}

In Norway, almost $80 \%$ of nursing home residents have dementia, and $90 \%$ have one or more neuropsychiatric symptoms. ${ }^{1}$ Neuropsychiatric symptoms vary in patients with dementia, and the prevalence of these symptoms differs according to the diagnosis and severity of the dementia. ${ }^{2}$ Non-pharmacological interventions are the treatments of choice for neuropsychiatric symptoms, but a pharmacological approach is recommended if these symptoms persist. ${ }^{4}$ In a review, Sink and colleagues identified four randomised controlled trials reporting the effect of antidepressants on neuropsychiatric symptoms, ${ }^{5}$ one study reported a significant effect after 12 weeks' treatment ${ }^{6}$ and the remaining studies were negative. A Cochrane review came to the same conclusion. ${ }^{7}$ A recent review summarised 19 randomised controlled trials comparing antidepressants with antipsychotic drugs or a placebo for neuropsychiatric symptoms in dementia. ${ }^{8}$ Eleven of these 19 studies recognised that antidepressants were effective in treating neuropsychiatric symptoms.

The effect of antidepressants in patients with dementia has been studied in many randomised controlled trials of varying quality, summarised in meta-analyses. ${ }^{9} 10$ These trials showed a trend towards antidepressants being effective, but the meta-analysis 
by Nelson and colleagues could not confirm the efficacy of antidepressants on depression in patients with dementia. ${ }^{9}$ Furthermore, a Cochrane review and a recent randomised controlled trial showed no effect of sertraline and mirtazepine over placebo on depression in dementia. ${ }^{10}{ }^{11}$

About $40 \%$ of residents in Norwegian nursing homes are prescribed antidepressants, ${ }^{1}$ of whom about $50 \%$ prescribed a selective serotonin reuptake inhibitor. However, the indication for the prescription is unclear. ${ }^{12}$ Although selective serotonin reuptake inhibitors have favourable side effect profiles, a profile was questioned by a recent study. ${ }^{13}$ Similar frequencies of psychotropic drug use in nursing homes have been found in other studies, ${ }^{14}$ and since antidepressants could be harmful to patients, the effect of withdrawing the drugs should be studied.

Therefore, we aimed to study the effects of discontinuing different selective serotonin reuptake inhibitors in relation to depressive symptoms, neuropsychiatric symptoms, and side effects in patients with dementia living in Norwegian nursing homes. Traditionally, discontinuation studies evaluate the effect of the discontinuation of treatment in successfully treated patients. In our study, we evaluated the effect of treatment discontinuation in patients in whom we did not know whether the treatment had any effect.

\section{Methods \\ Study design}

The study was a 25 week, double blind, parallel group, randomised, placebo controlled discontinuation trial of four selective serotonin reuptake inhibitors (escitalopram, citalopram, sertraline, and paroxetine), done in 52 nursing homes in Norway, independently of any pharmaceutical company. At the first week after baseline assessment, the antidepressants were either tapered off and replaced by a placebo (discontinuation group) or replaced by a study drug containing active medication with the same substance and same dose as before inclusion (continuation group). $\mathrm{H}$ Lundbeck $\mathrm{A} / \mathrm{S}$ provided the study with escitalopram tablets and placebos for $72(56 \%)$ patients. Encapsulated tablets for the remaining three antidepressants and corresponding placebo capsules containing only inert filler were purchased from Catalent Pharma Solutions, Bolton, United Kingdom.

The trial was registered in ClinicalTrial.gov on 3 January 2008 (NCT00594269).

\section{Randomisation and masking}

We used computer generated randomisation (1:1) in blocks of four. Packing of study treatment was done at the Hospital Pharmacy at the Innlandet Hospital Trust, and was kept hidden, by the use of blank labels, from the participants, caregivers, and the assessors until the completion of data collection and statistical analyses. Randomisation was done across study centres and facilities.

\section{Patients}

Participants were nursing home residents (men and women), recruited by 16 study centres in Norway. We included patients who were diagnosed with dementia in Alzheimer's disease, vascular dementia, or a mixture of Alzheimer's disease and vascular dementia (as defined by ICD-10 (international classification of diseases, 10th revision, diagnostic criteria for research); had been nursing home residents for more than four weeks; had a neuropsychiatric symptom; and were prescribed a selective serotonin reuptake inhibitor for at least three months.
Exclusion criteria included a clinical history of a depressive disorder or schizophrenia, severe somatic disease or terminal illness, or an inability to take tablets or capsules as prescribed. The exclusion of people with a documented depressive disorder was based on interviews with the nursing home doctors as well as studying the patients' medical records. Exclusion of patients with severe physical illness was based on ethical reasons and the assumption that they would not complete a study period of 25 weeks. No changes in the dose of the current antidepressant treatment were allowed in the last four weeks before inclusion and throughout the study period. Changes in the prescription of psychotropic drugs other than antidepressants during the study period were allowed. The participants (if competent) or their next of kin (legally representing if no kin) gave informed written consent.

\section{Assessments and outcomes}

The project leader (SB) and project coordinator taught research nurses about the study protocol and questionnaires in a one day training course. A geriatric psychiatrist or general practitioner trained in geriatric psychiatry studied the participants' medical records and, if necessary, examined the patient to ensure correct diagnosis of dementia and to ensure exclusion of patients with a depressive disorder. The research nurses collected all the data from the nursing homes, closely supervised by the project leader and the project coordinator to ensure a reliable data collection. We did efficacy assessments at baseline and at four, seven, 13, and 25 weeks. Primary endpoints were patients' scores on the Cornell scale for depression in dementia ${ }^{15}$ and the neuropsychiatric inventory ${ }^{16}$ after 25 weeks. The Cornell scale (score range 0-38) assesses the depressive symptoms of patients with dementia ( $\geq 9$ points is a sign of a depressive disorder; $\geq 13$ is a sign of a severe depressive disorder). ${ }^{17} \mathrm{We}$ divided the Cornell scale into two subscales: mood (sadness, anxiety, pessimism, suicidal thoughts, poor self esteem, and delusion) and non-mood (the remaining 13 symptoms), according to a recent Norwegian factor analysis of a large number of patients living in nursing homes. ${ }^{18}$

The neuropsychiatric inventory (10 item version) assesses neuropsychiatric symptoms and divides them into the following items: delusions, hallucinations, agitation, depression, anxiety, apathy, irritability, euphoria, disinhibition, and aberrant motor behaviour. For each item, it scores the frequency (1-4 points) and severity (1-3 points) of symptoms. The frequency and severity scores are then multiplied. A final score of 4 or more is regarded as a clinically relevant symptom, and a score of 9 or more is regarded as a severe symptom. ${ }^{19}$ In a recent published factor analysis of many nursing home patients, we divided the neuropsychiatric inventory into subsyndromes: affective (depression and anxiety), psychosis (hallucinations and delusions), agitation (agitation, irritability, and disinhibition), and apathy. ${ }^{20}$

The degree of dementia, cognitive impairment, extra pyramidal side effects, and the level of function were assessed at baseline and after 25 weeks with the clinical dementia rating scale, ${ }^{21}$ severe impairment battery, ${ }^{22}$ unified Parkinson disease rating scale (six item version), ${ }^{23}$ and Lawton and Brody's physical self maintenance scale, ${ }^{24}$ respectively. The clinical dementia rating scale is a six item questionnaire staging the severity of dementia: none (score 0 ), possible (0.5), mild (1), moderate (2), or severe (3). The severe impairment battery scale is a cognitive test with 51 items (score range 0-100), specifically developed for patients with moderate and severe dementia. 
Secondary endpoints were patients' scores after 25 weeks on the unified Parkinson's disease rating scale, quality of life-Alzheimer's disease scale, ${ }^{25}$ Lawton and Brody's physical self maintenance scale, severe impairment battery score, and clinical dementia rating scale. We used the severe impairment battery score unless patients were unable to communicate or refused testing. The scale for quality of life-Alzheimer's disease was used for both patients and caregivers. We used the additional assessments on one of the nurses at the nursing home.

\section{Statistical analysis}

Our power calculation was based on the only published pilot study on the discontinuation of antidepressant treatment in people with dementia. ${ }^{26}$ The pilot study included 11 patients using antidepressants, and all patients had their treatment withdrawn. Mean scores on the Cornell scale were 6.91 (standard deviation 4.48) at baseline and 3.25 (3.40) after 24 weeks without antidepressants (mean difference -3.66 , following a normal distribution). ${ }^{26} \mathrm{We}$ assumed that patients continuing with medication would have the same Cornell scale score as this pilot study, after 24 weeks from baseline, and calculated the sample size on the basis of a drop out of $60 \%$, as done in the pilot study. Assuming a mean score of 6.91 in the continuation group and a 3.66 point reduction to 3.25 in the discontinuation group, with power $80 \%, 5 \%$ significance level, two sided test, and standard deviation of 3.4, we needed a sample size of 18 patients per group in our study. To allow for a $60 \%$ drop out, we increased this number to 45 patients.

In the pilot study ${ }^{26}$ means scores on the neuropsychiatric inventory at baseline and after 24 weeks were 29.18 (standard deviation 20.19) and 17.25 (21.36), respectively (mean difference -11.93 , following a normal distribution). Since the pilot study had a high dropout rate, we attempted to increase the rate in our study by improving cooperation with the study centres and nursing homes. For the sample size calculation of these scores, we therefore chose a drop out of $33 \%$, which corresponds to the rates found in other discontinuation studies of nursing homes. Assuming a mean score of 29.18 in the continuation group and a decrease to 17.25 in the discontinuation group, with power $80 \%, 5 \%$ significance level, two sided test, and standard deviation of 21.36, we needed a sample size of 51 patients per group. To allow for a 33\% drop out, we increased this number to 76 patients. We had planned to enrol 152 patients in the study, but because the inclusion rate was too slow, and we included 128 patients.

We included all patients in the safety analysis, and all patients with at least one assessment after the baseline $(n=117)$ in the efficacy analysis. We analysed patients with complete data $(n=81)$ for changes in the primary and secondary endpoints using analysis of covariance, presented as observed cases. Assessment scales that lacked more than $20 \%$ of the data were not analysed. Analyses were done across study centres rather than for each centre, because ten centres were small $(<10$ patients each).

We did statistical analyses using SPSS, version 15.0. The baseline characteristics were analysed with the independent student's $t$ test for parametric data, Mann-Whitney U test for non-parametric data, and $\chi^{2}$ statistics or Fischer's exact test for categorical data. We compared the two study groups using a linear multi level model analyses for the difference between baseline and 25 weeks for the two primary outcomes (Cornell scale and neuropsychiatric inventory).

We did subgroup analyses for patients with a Cornell scale score less than 8 and those with a score of 8 and above by linear multi level model analysis; we also analysed subscores of the neuropsychiatric inventory and Cornell scale. Categorisation of subscores of the both scales was based on previous studies, ${ }^{18} 20$ but we decided to include these analyses only after study initiation. We used McNemar's test to analyse the number of patients who changed from a Cornell scale score of 13 or lower at baseline to a score higher than 13 at 25 weeks, and vice versa.

\section{Results Study population}

Patients were included from August 2008 to June 2010. After assessing 205 patients for eligibility, we excluded 77 for various reasons (fig $\downarrow$ ). We assigned 63 patients to discontinue antidepressant treatment and 65 to continue with treatment. Of 128 patients included, 72 (56\%) used escitalopram, 47 (37\%) citalopram, five (4\%) sertraline, and four (3\%) paroxetine, which represented the distribution of antidepressant treatment in Norwegian nursing homes. We did not do subgroup analyses of different antidepressants because we wanted to study the general effect of discontinuation rather than analyse a specific selective serotonin reuptake inhibitor, and also because some subgroups of prescribed drugs were small.

We did not see any significant differences between the two groups at baseline, in terms of sex, age, the clinical dementia rating scale, dementia diagnosis, the Cornell scale, or the neuropsychiatric inventory (table $1 \Downarrow$ ). Mean age was 85.3 years for the discontinuation group and 86.1 years for the continuation group. The proportion of women in the discontinuation and continuation groups was $78 \%$ and $72 \%$, respectively. At baseline, the discontinuation and continuation groups had a median score on the Cornell scale of 4 (interquartile range 1.5-6.5) and 5 (1.5-8.5), respectively. Median scores for the neuropsychiatric inventory at baseline were $13(3.5-22.5)$ and 16 (6.5-25.5) for the discontinuation and continuation groups, respectively.

We excluded 11 patients (four in the discontinuation group and seven in the continuation group) from the efficacy analyses, either because no post baseline assessments were available or because of study protocol violation; therefore, we included 117 patients for the efficacy analysis. Forty seven (37\%) patients withdrew from the study prematurely, $28(44 \%)$ in the discontinuation group and $19(29 \%)$ in the continuation group. The only reason for drop outs that differed significantly between groups was an increase in neuropsychiatric symptoms, for 13 (21\%) patients in the discontinuation group and four $(6 \%)$ in the continuation group (table $2 \Downarrow$ ).

\section{Efficacy analyses}

Table $3 \Downarrow$ presents the results for the complete cases. The total score on the Cornell scale at 25 weeks was 6.03 (standard deviation 4.76) for the discontinuation group and 4.42 (3.77) for the continuation group $(\mathrm{P}=0.045)$. Corresponding values for total neuropsychiatric inventory scores were 22.54 (18.58) and 14.74 (9.15), respectively $(\mathrm{P}=0.056)$. For the secondary endpoints, we saw no significant differences between the two groups after 25 weeks.

Table $4 \Downarrow$ presents the results of the linear multi level model (mixed model) analyses. After 25 weeks, the regression coefficient representing the difference in total score of the Cornell scale was -2.89 (95\% confidence interval -4.76 to -1.02 ; $\mathrm{P}=0.003$ ); a negative score indicates a higher score in the discontinuation group. We also saw a similar result between the groups for total scores in the neuropsychiatric inventory, 
but this difference was non-significant $(-5.96(-12.35$ to -0.44$)$; $\mathrm{P}=0.068$ ).

Sensitivity analysis of patients with a baseline score of $0-8$ on the Cornell scale showed an increased score in the

discontinuation group compared with the continuation group (difference -3.36 (95\% confidence interval -5.23 to -1.48 ),

$\mathrm{P}=0.001$ ). For patients with a baseline score on the Cornell scale higher than 8 , those in the discontinuation group had a non-significant decrease in score after 25 weeks compared with those in the continuation group (2.20 (-1.68 to 6.09), $\mathrm{P}=0.251$ ). When analysing the mood and non-mood components of the Cornell scale separately, the discontinuation group had an increased score compared with the continuation group for both subscales. However, this difference was significant only for the non-mood subscale $(-1.89$ ( -3.56 to -0.21$) ; \mathrm{P}=0.028)$. We also saw a significant difference in depressive symptoms after 25 weeks in the affective subsyndrome of the neuropsychiatric inventory $(-2.25(-4.24$ to -0.27$) ; \mathrm{P}=0.027)$.

In the continuation group, two (3\%) patients with a baseline score of 0-13 on the Cornell scale scored 14 points and higher after 25 weeks, and two (3\%) with a baseline score of 14 and higher scored $0-13$ points after 25 weeks. Fifty four (90\%) patients continued to score $0-13$ points at baseline and after 25 weeks, and two (3\%) continued to score 14-39 points at baseline and after 25 weeks $(\mathrm{P}=1.00)$.

For the discontinuation group, eight (14\%) patients switched from scoring 0-13 points on the Cornell scale at baseline to scoring 14 and higher points at 25 weeks. No patients switched from baseline scores of 14 and higher to scores of 0-13 at 25 weeks. Forty eight (83\%) patients continued to score $0-13$ points at baseline and after 25 weeks, and two (3\%) continued to score 14 or higher points both at baseline and after 25 weeks $(\mathrm{P}=0.008)$.

\section{Tolerability and safety}

We saw no significant differences between the groups in scores for the unified Parkinson's disease rating scale after 25 weeks (discontinuation group 3.63 (standard deviation 3.32) $v$ continuation group 3.76 (3.89); $\mathrm{P}=0.377$ ). Twenty eight (44\%) patients in the discontinuation group and $19(21 \%)$ in the continuation group withdrew from the study prematurely (table 2 ). Thirteen $(20 \%)$ patients in the discontinuation group and four $(6 \%)$ in the continuation group withdrew from the study prematurely because of increased depressive or neuropsychiatric symptoms $(\mathrm{P}=0.019$; table 2$)$.

\section{Discussion}

\section{Main findings}

To our knowledge, we have published the first randomised controlled trial on antidepressant discontinuation in patients with dementia. Previous studies on antidepressant treatment for neuropsychiatric symptoms in dementia have reported against antidepressants, and our hypothesis was that discontinuing treatment would not change patients' depressive symptoms. ${ }^{26}$ In our study, patients in the discontinuation group had a significant increase in their depressive symptoms compared with those in the continuation group, as shown in the Cornell scale of depression in dementia (total score, non-mood subscore), the affective subscore of the neuropsychiatric inventory, response analyses (worsening or improving by $>30 \%$ ), and the proportion of patients changing from a low to high score on the Cornell scale after 25 weeks' intervention. Additionally, between group differences in the total score of the neuropsychiatric inventory was nearly significant. We did not see any significant differences in the secondary endpoints between the groups.

\section{Strengths and weaknesses of the study}

A strength of our study was its multicentre, randomised, double blind, placebo controlled design, which controlled for all covariate variables. We used internationally recommended assessment tools, with 25 week follow-up. We used independent monitoring nurses to supervise the study. The main finding in our study was confirmed by different statistical analyses. Our study findings were also confirmed by two different assessment tools.

The main limitation of our study was the uncertainty of whether the patients had a depressive disorder at the initiation of the antidepressant drug. Although we made substantial efforts to exclude patients with a documented depressive disorder, inclusion of patients with a depressive disorder could have biased the results. Furthermore, the study had a high dropout rate; only $63 \%$ of patients completed the 25 week study period. However, study withdrawals owing to increased neuropsychiatric symptoms were more common in the discontinuation group than in the continuation group, which could indicate that discontinuation was unfavourable. The study involved many research nurses, which could have biased the data even though each research nurse had completed a one day course. The discontinuation of antidepressant treatment over one week was fairly quick, and some of the patients could have had discontinuation symptoms rather than increased depressive symptoms. However, discontinuation symptoms should not have been present after 25 weeks.

\section{Strengths and weaknesses in relation to other studies}

Some caution should be taken in the interpretation of our findings, because our cohort is different from previous studies. In our study, we excluded patients who had a depressive disorder (according to ICD-10 criteria) either at inclusion or documented in their medical records, even though randomised controlled trials investigating the initiation of antidepressant treatment have often included patients with a depressive disorder.

Five $(8 \%)$ patients in the continuation group and two (3\%) in the discontinuation group had a score higher than 13 points on the Cornell scale at baseline, which could indicate a severe depression. ${ }^{17}$ Nevertheless, these patients were clinically judged not to have a depressive disorder at inclusion. Although the indication for treatment with antidepressants is unclear for patients enrolled in our study, most of them had been prescribed antidepressants for depressive symptoms or apathy after their dementia.

The focus on cerebrovascular side effects of antipsychotic drugs in the past year has reduced the prescription of antipsychotic drugs for neuropsychiatric symptoms in Norway, and more patients could have been prescribed antidepressant for neuropsychiatric symptoms. The aim of our study was to evaluate the effect of antidepressants on depressive symptoms and neuropsychiatric symptoms, and we took care to ensure that the enrolled patients did not have a depressive disorder. Because the patients' depressive symptoms worsened after discontinuation, we concluded that antidepressants could be effective in treating depressive symptoms in patients with dementia. In the discontinuation group of complete cases, the mean Cornell scale score increased from 5.03 to 6.03, which still is under the cutoff score that is usually used to define a 
possible depressive disorder in patients with dementia. ${ }^{15}{ }^{17}$ The mean change of 1.0 was a $20 \%$ worsening from baseline, but was this change clinically significant? We did not find statistically significant changes on quality of life, activity of daily life function, or side effects.

Our results suggest that the changes in the Cornell score and neuropsychiatric inventory were driven by the effect of selective serotonin reuptake inhibitors on mood symptoms rather than on neuropsychiatric symptoms. Our findings are supported by previous randomised controlled trials on the effect of antidepressants on neuropsychiatric symptoms of dementia in patients with Alzheimer's disease, which have shown a benefit of antidepressants over placebo on emotional bluntness, anxiety, depressive mood, and restlessness $;{ }^{27}$ a favourable effect on behavioural symptoms; ${ }^{6}$ and a decreased frequency of irritability and apathy. ${ }^{28}$ Previous trials have also shown antidepressants to be as effective in reducing agitation in dementia as haloperidol, ${ }^{29}$ and to have a similar effect on behavioural symptoms and psychosis as risperidone.$^{30}$ Nevertheless, both meta-analyses and systematic reviews have concluded that little evidence is available to show the effectiveness of antidepressants on neuropsychiatric symptoms. ${ }^{78}$

The favourable effect of antidepressants on depression has also been shown in patients with Alzheimer's disease and severe depression, ${ }^{31}$ but most trials have shown negative results. ${ }^{11} 3233$ A meta-analysis of the effect of antidepressants on depression in patients with dementia concluded that the evidence in favour of using antidepressants in treating depression in patients with dementia was weak. ${ }^{10}$ In our study, most of the patients remained unchanged after 25 weeks, as shown with a McNemar analysis of the proportion of patients changing from a low to a high score on the Cornell scale. These findings could indicate that discontinuation of antidepressants is safe in most of patients with dementia.

\section{Conclusions and policy implications}

In our study, patients with discontinued treatment showed a significant increase in depressive symptoms and those who continued antidepressant treatment had a small decrease in such symptoms after 25 weeks. A worsening of depressive symptoms after discontinuation could indicate that antidepressants are effective in patients with dementia and neuropsychiatric symptoms. Nevertheless, $86 \%$ of the patients in the discontinuation group tolerated the discontinuation because they remained in the same subscore group ( $0-13$ and $\geq 14$ points) of the Cornell scale of depression in dementia. Consequently, although antidepressants could be discontinued in most patients with dementia, these patients should be monitored carefully to identify those with worsening depressive symptoms.

Contributors: SB, GS, and KE participated in design of the study. SB was principal study investigator and drafted the manuscript. All authors participated in analysis and interpretation of results, critical revision of the article for intellectual content, and approved the final version of the manuscript for publication. SB, GS, and KE are the guarantors of the paper.

Funding: The study was funded by unrestricted grants from the Innlandet Hospital Trust, the Research Council of Norway, and the South-Eastern Norway Regional Health Authority.

Conflicts of interest: All authors have completed the ICMJE uniform disclosure form at www.icmje.org/coi_disclosure.pdf (available on request from the corresponding author) and declare: SB received study treatment free of charge from $\mathrm{H}$ Lundbeck $\mathrm{A} / \mathrm{S}$ (escitalopram tablets and placebo), but with no obligations for publication, and the study received funding support from the Innlandet Hospital Trust, the Research Council of Norway, and the South-Eastern Norway Regional Health Authority; no financial relationships with any organisations that might have an interest in the submitted work in the previous 3 years; no other relationships or activities that could appear to have influenced the submitted work.

Ethical approval: The study was approved by the Norwegian Medicines Agency, Regional Committee of Medical Research Ethics (2.2007.137), and Norwegian Directorate of Health. Approval was conducted according to the standard of good clinical practice, and monitored independently by staff members from Oslo University Hospital. The procedures were conducted in accordance with the Helsinki Declaration as revised in 1983.

Patient consent: Participants (if competent) or their next of kin (or legal representative if no kin) provided informed written consent.

Data sharing: Technical appendix, statistical code, and dataset are available from the corresponding author. A full trial protocol can be accessed at www.sykehuset-innlandet.no/omoss/avdelinger/ alderpsykiatrisk-forskningssenter/prosjekter/Sider/desep.aspx.

1 Selbaek G, Kirkevold O, Engedal K. The prevalence of psychiatric symptoms and behavioural disturbances and the use of psychotropic drugs in Norwegian nursing homes. Int J Geriatr Psychiatry 2007;222:843-9.

2 Wetzels R, Zuidema S, Jansen I, Verhey F, Koopmans R. Course of neuropsychiatric symptoms in residents with dementia in long-term care institutions: a systematic review. Int Psychogeriatr 2010;22:1040-53.

3 Selbaek G, Kirkevold O, Engedal K. The course of psychiatric and behavioral symptoms and the use of psychotropic medication in patients with dementia in Norwegian nursing homes-a 12-month follow-up study. Am J Geriatr Psychiatry 2008;16:528-36.

4 Ballard C, Corbett A. Management of neuropsychiatric symptoms in people with dementia. CNS Drugs 2010;24:729-39.

5 Sink KM, Holden KF, Yaffe K. Pharmacological treatment of neuropsychiatric symptoms of dementia: a review of the evidence. JAMA 2005;293:596-608.

6 Pollock BG, Mulsant BH, Rosen J, Sweet RA, Mazumdar S, Bharucha A, et al. Comparison of citalopram, perphenazine, and placebo for the acute treatment of psychosis and behavioral disturbances in hospitalized, demented patients. Am J Psychiatry 2002;159:460-5.

7 Seitz DP, Adunuri N, Gill SS, Gruneir A, Herrmann N, Rochon P. Antidepressants for agitation and psychosis in dementia. Cochrane Database Syst Rev 2011;2:CD008191.

8 Henry G, Williamson D, Tampi RR. Efficacy and tolerability of antidepressants in the treatment of behavioral and psychological symptoms of dementia, a literature review of evidence. Am J Alzheimers Dis Other Demen 2011;26:169-83.

9 Nelson JC, Devanand DP. A systematic review and meta-analysis of placebo-controlled antidepressant studies in people with depression and dementia. J Am Geriatr Soc 2011;59:577-85.

10 Bains J, Birks JS, Dening TR. The efficacy of antidepressants in the treatment of depression in dementia. Cochrane Database Syst Rev 2002;4:CD003944.

11 Banerjee S, Hellier J, Dewey M, Romeo R, Ballard C, Baldwin R, et al. Sertraline or mirtazapine for depression in dementia (HTA-SADD): a randomised, multicentre, mirtazapine for depression in dementia (HTA-SADD): a randomisect

12 Barca ML, Selbaek G, Laks J, Engedal K. Factors associated with depression in Norwegian nursing homes. Int $J$ Geriatr Psychiatry 2009;24:417-25.

13 Coupland C, Dhiman P, Morriss R, Arthur A, Barton G, Hippisley-Cox J. Antidepressant use and risk of adverse outcomes in older people: population based cohort study. BMJ 2011;343:d4551.

14 Wetzels RB, Zuidema SU, de Jonghe JF, Verhey FR, Koopmans RT. Course of neuropsychiatric symptoms in residents with dementia in nursing homes over 2-year period. Am J Geriatr Psychiatry 2010;18:1054-65.

15 Alexopoulos GS, Abrams RC, Young RC, Shamoian CA. Cornell scale for depression in dementia. Biol Psychiatry 1988;23:271-84.

16 Cummings JL, Mega M, Gray K, Rosenberg-Thompson S, Carusi DA, Gornbein J. The Neuropsychiatric Inventory: comprehensive assessment of psychopathology in dementia. Neurology 1994;44:2308-14.

17 Barca ML, Engedal K, Selbaek G. A reliability and validity study of the cornell scale among elderly inpatients, using various clinical criteria. Dement Geriatr Cogn Disord 2010;29:438-47.

18 Barca ML, Selbaek G, Laks J, Engedal K. The pattern of depressive symptoms and factor analysis of the Cornell Scale among patients in Norwegian nursing homes. Int $J$ Geriatr Psychiatry 2008;23:1058-65.

19 Steinberg M, Tschanz JT, Corcoran C, Steffens DC, Norton MC, Lyketsos CG, et al. The persistence of neuropsychiatric symptoms in dementia: the Cache County Study. Int $J$ Geriatr Psychiatry 2004;19:19-26.

20 Selbaek G, Engedal K. Stability of the factor structure of the Neuropsychiatric Inventory in a 31-month follow-up study of a large sample of nursing-home patients with dementia. Int Psychogeriatr 2012;24:62-73.

21 Berg L. Clinical dementia rating (CDR). Psychopharmacol Bull 1988;24:637-9.

22 Panisset M, Roudier M, Saxton J, Boller F. Severe impairment battery. A neuropsychological test for severely demented patients. Arch Neurol 1994;51:41-5.

23 Ballard C, McKeith I, Burn D, Harrison R, O'Brien J, Lowery K, et al. The UPDRS scale as a means of identifying extrapyramidal signs in patients suffering from dementia with Lewy bodies. Acta Neurol Scand 1997;96:366-71.

24 Lawton MP, Brody EM. Assessment of older people: self-maintaining and instrumental activities of daily living. Gerontologist 1969;9:179-86. 


\section{What is already known on this topic}

Psychotropic drugs are frequently prescribed to patients with dementia

Evidence is weak in relation to the pharmacological treatment of neuropsychiatric symptoms and depressive symptoms in patients with dementia

\section{What this study adds}

Discontinuation of escitalopram, citalopram, sertraline, or paroxetin in patients with dementia led to a significant increase in their depressive symptoms

Significantly more patients who discontinued treatment than those continuing treatment withdrew from the study prematurely, owing to increased neuropsychiatric symptoms

Discontinuation of antidepressants was tolerated in most study patients

25 Thorgrimsen L, Selwood A, Spector A, Royan L, de Madariaga LM, Woods RT, et al. Whose quality of life is it anyway? The validity and reliability of the Quality of Life-Alzheimer's Disease (QoL-AD) scale. Alzheimer Dis Assoc Disord 2003;17:201-8.

26 Bergh S, Engedal K. The withdrawal of antipsychotics and antidepressants from patients with dementia and BPSD living in nursing homes: an open pilot study. Int J Geriatr Psychiatry 2008;23:877-9.

27 Nyth AL, Gottfries CG. The clinical efficacy of citalopram in treatment of emotional disturbances in dementia disorders. A Nordic multicentre study. Br J Psychiatry 1990;157:894-901.

28 Siddique H, Hynan LS, Weiner MF. Effect of a serotonin reuptake inhibitor on irritability, apathy, and psychotic symptoms in patients with Alzheimer's disease. J Clin Psychiatry 2009;70:915-8.

29 Gaber S, Ronzoli S, Bruno A, Biagi A. Sertraline versus small doses of haloperidol in the treatment of agitated behavior in patients with dementia. Arch Gerontol Geriatr Suppl 2001;7:159-62.

30 Pollock BG, Mulsant BH, Rosen J, Mazumdar S, Blakesley RE, Houck PR, et al. A double-blind comparison of citalopram and risperidone for the treatment of behavioral and psychotic symptoms associated with dementia. Am J Geriatr Psychiatry 2007:15:942-52.
31 Lyketsos CG, DelCampo L, Steinberg M, Miles Q, Steele CD, Munro C, et al. Treating depression in Alzheimer disease: efficacy and safety of sertraline therapy, and the benefits of depression reduction: the DIADS. Arch Gen Psychiatry 2003;60:737-46.

32 Rosenberg PB, Drye LT, Martin BK, Frangakis C, Mintzer JE, Weintraub D, et al. Sertraline for the treatment of depression in Alzheimer disease. Am J Geriatr Psychiatry 2010;18:136-45.

33 Weintraub D, Rosenberg PB, Drye LT, Martin BK, Frangakis C, Mintzer JE, et al. Sertraline for the treatment of depression in Alzheimer disease: week-24 outcomes. Am J Geriatr Psychiatry 2010;18:332-40.

Accepted: 24 January 2012

\section{Cite this as: BMJ 2012;344:e1566}

This is an open-access article distributed under the terms of the Creative Commons Attribution Non-commercial License, which permits use, distribution, and reproduction in any medium, provided the original work is properly cited, the use is non commercial and is otherwise in compliance with the license. See: http://creativecommons.org/licenses/bync/2.0/ and http://creativecommons.org/licenses/by-nc/2.0/legalcode. 


\section{Tables}

\section{Table 1| Baseline characteristics}

\begin{tabular}{|c|c|c|}
\hline & Discontinued treatment $(n=63)$ & Continued treatment $(n=65)$ \\
\hline Age (years, mean) ${ }^{*}$ & $85.3(8.2)$ & $86.1(6.7)$ \\
\hline Female sex (no of patients $(\%)) \dagger$ & $49(78)$ & $47(72)$ \\
\hline \multicolumn{3}{|l|}{ Diagnosis (no of patients $(\%)) \dagger$} \\
\hline Alzheimer's disease & $32(51)$ & $38(58)$ \\
\hline Vascular dementia & $16(25)$ & $10(15)$ \\
\hline Mixed (Alzheimer's disease and vascular dementia) & $15(24)$ & $17(26)$ \\
\hline \multicolumn{3}{|l|}{ Clinical dementia rating scale (no of patients $(\%)) \dagger$} \\
\hline 1 point & $8(13)$ & $8(12)$ \\
\hline 2 points & $32(51)$ & $31(48)$ \\
\hline 3 points & $23(37)$ & $26(40)$ \\
\hline \multicolumn{3}{|l|}{ Severe impairment battery } \\
\hline Median score $\ddagger$ & $78(64.5-91.5), n=50$ & $81(68-94), n=52$ \\
\hline Mean score & $72.5(19.5)$ & $70.9(25.6)$ \\
\hline \multicolumn{3}{|l|}{ Cornell scale } \\
\hline Median score $\ddagger$ & $4(1.5-6.5), n=61$ & $5(1.5-8.5), n=64$ \\
\hline Mean score & $5.03(4.15)$ & $5.89(4.62)$ \\
\hline \multicolumn{3}{|l|}{ Cornell scale (categorised) (no of patients (\%)) } \\
\hline $0-8$ points & $50(81), n=62$ & $44(69), n=64$ \\
\hline 9-13 points & $10(16)$ & $15(23)$ \\
\hline$\geq 14$ points & $2(3)$ & $5(8)$ \\
\hline \multicolumn{3}{|l|}{ Cornell scale (mood) } \\
\hline Median scoreł & $1(0-2)$ & $1(-1-3), n=64$ \\
\hline Mean score & $1.28(1.71)$ & $1.95(2.40)$ \\
\hline \multicolumn{3}{|l|}{ Cornell scale (non-mood) } \\
\hline Median scoreł & $3(1-5), n=62$ & $2.5(0-5), n=64$ \\
\hline Mean score & $3.44(3.30)$ & $3.32(2.90)$ \\
\hline \multicolumn{3}{|l|}{ Neuropsychiatric inventory } \\
\hline Median scoreł & $13(3.5-22.5)$ & $16(6.5-25.5)$ \\
\hline Mean score & $17.78(16.75)$ & $17.63(14.09)$ \\
\hline \multicolumn{3}{|l|}{ Lawton and Brody's physical self maintenance scale } \\
\hline Median scoreł & $18(14.5-21.5)$ & $19(9-29)$ \\
\hline Mean score & $17.25(4.67)$ & $18.14(5.45)$ \\
\hline \multicolumn{3}{|l|}{ Quality of life-Alzheimer's disease scale } \\
\hline Patients' rating (mean) ${ }^{*}$ & $33.67(5.03), \mathrm{n}=46$ & $34.38(5.05), n=50$ \\
\hline Caregivers' rating (mean) ${ }^{*}$ & $31.06(5.57)$ & $30.72(5.05)$ \\
\hline \multicolumn{3}{|l|}{ Unified Parkinson's disease rating scale } \\
\hline Median scoreł & $2(0-4)$ & $3(0.5-5.5)$ \\
\hline Mean score & $3.08(2.84)$ & $4.38(4.06)$ \\
\hline Weight $(\mathrm{kg}, \text { mean })^{*}$ & $66.46(15.06), n=54$ & $66.33(11.78), \mathrm{n}=58$ \\
\hline \multicolumn{3}{|l|}{ No of other psychotropic drugs taken } \\
\hline Median $\ddagger$ & $2(1.5-2.5)$ & $2(1-3)$ \\
\hline Mean & $1.94(1.01)$ & $2.11(1.11)$ \\
\hline \multicolumn{3}{|l|}{ No of falls/day in last 21 days before inclusion } \\
\hline Median $\neq$ & $0(0-0)$ & $0(0-0), n=64$ \\
\hline Mean & $0.02(0.09)$ & $0.01(0.03)$ \\
\hline
\end{tabular}


Table 1 (continued)

\begin{tabular}{lcc} 
& Discontinued treatment $(\mathbf{n}=63)$ & Continued treatment $(\mathrm{n}=65)$ \\
Median $\ddagger$ & $0(0-0)$ & $0(0-0)$ \\
\hline Mean & $0.67(3.63)$ & $0.18(0.55)$ \\
\hline
\end{tabular}

Data are median (interquartile range) or mean (standard deviation) unless stated otherwise. *Independent student's $t$ test. †Pearson $x^{2}$ test.

$\ddagger$ Mann-Whitney U test. 
Table 2| No (\%) of patients completing 25 week intervention and discontinuing intervention prematurely

\begin{tabular}{lccc} 
& Discontinued treatment $(\mathbf{n}=63)$ & Continued treatment (n=65) & $\mathbf{P}$ \\
\hline Completed 25 weeks intervention*) & $35(56)$ & $46(71)$ & 0.074 \\
\hline Discontinued intervention† & & & \\
\hline Death, somatic disease, or admitted to hospital & $7(11)$ & $7(11)$ & 1.000 \\
\hline Witten consent withdrawn & $5(8)$ & $4(6)$ & 0.742 \\
\hline Increased neuropsychiatric symptoms & $13(21)$ & $4(6)$ & $0.019 \neq$ \\
\hline Could not swallow study capsules & $2(3)$ & $1(2)$ & 0.616 \\
\hline Moved from nursing home & $1(2)$ & $1(2)$ & 1.000 \\
\hline Did not take study treatment as prescribed & 0 & $2(3)$ & 0.496 \\
\hline
\end{tabular}

*Pearson's $x^{2}$ test.

†Fisher's exact test.

$\ddagger P<0.05$. 


\begin{tabular}{|c|c|c|c|}
\hline & Discontinued treatment $(n=35)$ & Continued treatment $(n=46)$ & $\mathbf{P}$ \\
\hline \multicolumn{4}{|l|}{ Primary efficacy analysis } \\
\hline Cornell scale, total score & $6.03(4.76), \mathrm{n}=31$ & $4.42(3.77)$ & $0.045^{\star}$ \\
\hline Neuropsychiatric inventory, total score & $22.54(18.58)$ & $14.74(9.15)$ & 0.056 \\
\hline \multicolumn{4}{|l|}{ Secondary efficacy variables } \\
\hline Cornell scale, mood score & $1.43(1.69), \mathrm{n}=28$ & $1.49(1.68) n=43$ & 0.804 \\
\hline Cornell scale, non-mood score & $4.21(3.39) n=28$ & $2.80(2.35) n=40$ & 0.062 \\
\hline \multicolumn{4}{|l|}{ Cornell scale, responders $(\%) \dagger$} \\
\hline$>30 \%$ improvement & $13(22)$ & $19(33)$ & 0.193 \\
\hline $30 \%$ improvement to $30 \%$ deterioration & $14(24)$ & $22(38)$ & 0.096 \\
\hline$>30 \%$ deterioration & $32(54)$ & $17(29)$ & $0.006^{\star}$ \\
\hline Neuropsychiatric inventory, affective subsyndrome & $4.34(6.18)$ & $2.96(3.33)$ & 0.333 \\
\hline \multicolumn{4}{|c|}{ Neuropsychiatric inventory, affective subsyndrome, responders $(\%) \dagger$} \\
\hline$>30 \%$ improvement & $18(31)$ & $18(31)$ & 0.951 \\
\hline $30 \%$ improvement to $30 \%$ deterioration & $28(48)$ & $34(59)$ & 0.226 \\
\hline$>30 \%$ deterioration & $13(22)$ & $6(10)$ & 0.087 \\
\hline Neuropsychiatric inventory, agitation subsyndrome & $10.09(9.90)$ & $6.37(5.47)$ & 0.268 \\
\hline Neuropsychiatric inventory, psychotic subsyndrome & $2.54(5.02)$ & $1.57(2.65)$ & 0.360 \\
\hline Neuropsychiatric inventory, apathy & $0.29(0.468)$ & $0.41(0.50)$ & 0.216 \\
\hline \multicolumn{4}{|l|}{ Clinical dementia rating, score $(\%) \dagger$} \\
\hline Missing & $3(5)$ & $7(12)$ & 0.177 \\
\hline 0.5 & 0 & $1(2)$ & 0.311 \\
\hline 1 & $6(10)$ & $7(12)$ & 0.744 \\
\hline 2 & $23(39)$ & $19(33)$ & 0.483 \\
\hline 3 & $27(46)$ & $24(41)$ & 0.633 \\
\hline Severe impairment battery, score & 73.35 (23.96), $\mathrm{n}=23$ & $67.97(30.91), \mathrm{n}=37$ & 0.956 \\
\hline Unified Parkinson's disease rating scale, score & $3.63(3.32)$ & $3.76(3.89)$ & 0.377 \\
\hline Lawton and Brody's physical self maintenance scale, score & $18.46(5.59)$ & $18.11(5.38)$ & 0.915 \\
\hline Weight (kg) & $62.62(13.78), n=26$ & $66.67(11.47), \mathrm{n}=42$ & 0.092 \\
\hline Quality of life-Alzheimer's disease scale, patients' rating & $32.80(7.12), \mathrm{n}=20$ & $35.87(4.95), n=31$ & 0.314 \\
\hline Quality of life-Alzheimer's disease scale, caregivers' rating & $29.89(6.57)$ & $29.11(5.12), \mathrm{n}=45$ & 0.674 \\
\hline Change in no of falls/day in last 21 days before inclusion & $0.01(0.02)$ & $0.01(0.03), \mathrm{n}=45$ & 0.270 \\
\hline Oxazepam (mg)/day taken in last 21 days before inclusion & $0.17(0.38), n=34$ & $0.26(0.69)$ & 0.293 \\
\hline Change in no of psychotropic drugs taken & $1.77(0.97)$ & $1.87(1.00)$ & 0.170 \\
\hline \multicolumn{4}{|c|}{$\begin{array}{l}\text { Data are mean (standard deviation) and are calculated by analyses of covariance (with baseline values as covariate) unless stated otherwise. } \\
{ }^{*} P<0.05 \text {. }\end{array}$} \\
\hline
\end{tabular}


Table 4| Differences in primary outcomes between discontinuation and continuation study groups, after 25 weeks

\begin{tabular}{lcccc} 
Primary outcome & Regression coefficient* & Standard error & P & \\
Cornell scale & & & $95 \% \mathbf{C l}$ \\
\hline Total score & -2.89 & 0.94 & $0.003 \dagger$ & -4.76 to -1.02 \\
\hline Total score (baseline score $<9)$ & -3.36 & 0.94 & $0.001 \dagger$ & -5.23 to -1.48 \\
\hline Total score (baseline score $\geq 9)$ & 2.20 & 1.86 & 0.251 & -1.68 to 6.09 \\
\hline Mood score & -0.73 & 0.37 & 0.054 & -1.47 to 0.01 \\
\hline Non-mood score & -1.89 & 0.85 & $0.028 \dagger$ & -3.56 to -0.21 \\
\hline Neuropsychiatric inventory & & & & -12.35 to 0.44 \\
\hline Total score & -5.96 & 3.23 & 0.068 & -4.24 to -0.27 \\
\hline Affective subsyndrome & -2.25 & 1.00 & $0.027 \dagger$ & -3.93 to 1.64 \\
\hline Agitation subsyndrome & -1.14 & 1.40 & 0.417 & -2.32 to 1.19 \\
\hline Psychosis subsyndrome & -0.56 & 0.89 & 0.527 & -1.90 to 0.94 \\
\hline Apathy & -0.48 & 0.72 & 0.507 & \\
\hline
\end{tabular}

Data calculated by linear multi level model analysis.

${ }^{*}$ Negative score indicates higher score on Cornell scale and neuropsychiatric inventory in the discontinuation group.

$\dagger \mathrm{P}<0.05$. 


\section{Figure}

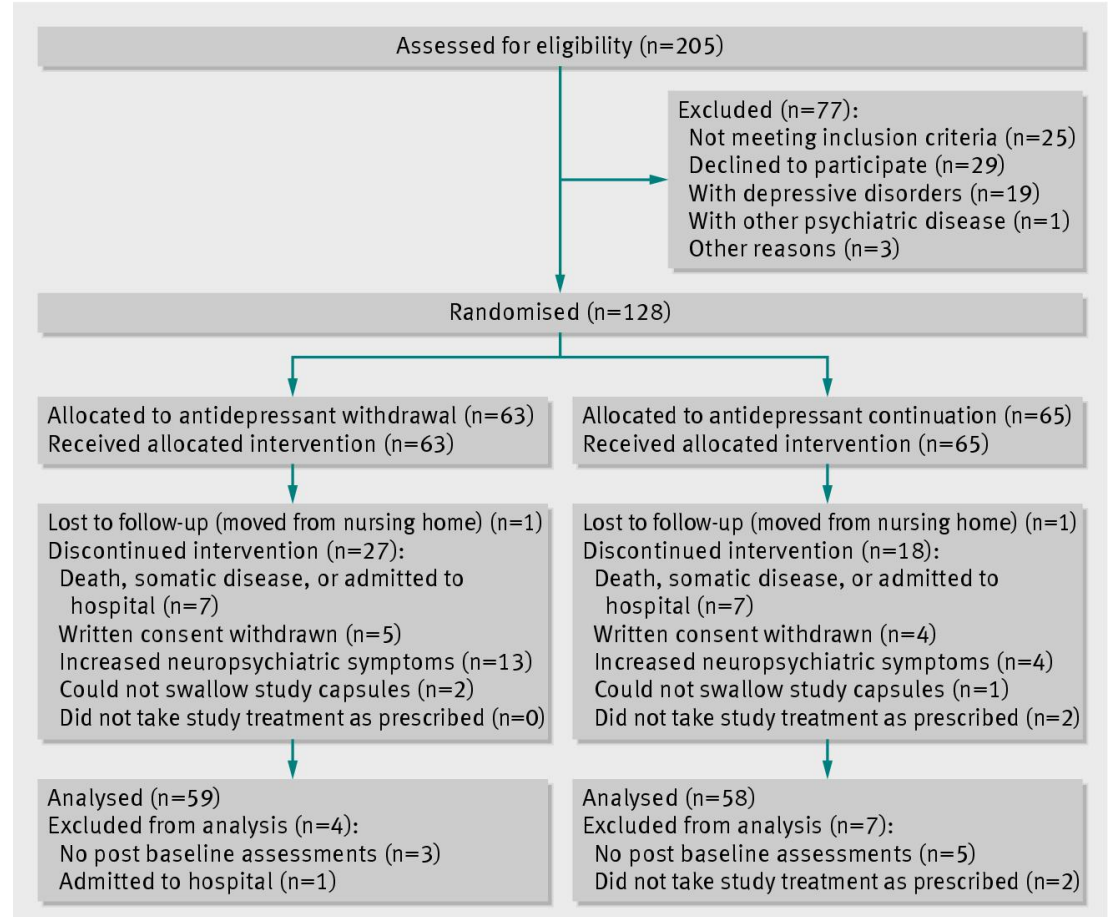

Study flowchart 